\title{
Magnetic nanoparticles with bulklike properties (invited)
}

\author{
Xavier Batlle, ${ }^{1, a)}$ N. Pérez, ${ }^{1}$ P. Guardia, ${ }^{1}$ O. Iglesias, ${ }^{1}$ A. Labarta, ${ }^{1}$ F. Bartolomé,${ }^{2}$ \\ L. M. García, ${ }^{2}$ J. Bartolomé, ${ }^{2}$ A. G. Roca, ${ }^{3}$ M. P. Morales, ${ }^{3}$ and C. J. Serna ${ }^{3}$ \\ ${ }^{1}$ Dep. Física Fonamental and Institut de Nanociència i Nanotecnologia (IN2UB), Universitat de Barcelona, \\ Martí i Franquès 1, 08028 Barcelona, Spain \\ ${ }^{2}$ Instituto de Ciencia de Materiales de Aragón (ICMA)-CSIC and Dep. Física Materia Condensada, \\ Universidad de Zaragoza, 50009 Zaragoza, Spain \\ ${ }^{3}$ Instituto de Ciencia de Materiales de Madrid (ICMM)-CSIC, 28049 Madrid, Spain
}

(Presented 18 November 2010; received 17 September 2010; accepted 30 November 2010; published online 4 April 2011)

\begin{abstract}
The magnetic behavior of $\mathrm{Fe}_{3-x} \mathrm{O}_{4}$ nanoparticles synthesized by either high-temperature decomposition of an organic iron precursor or low-temperature coprecipitation in aqueous conditions is compared. Transmission electron microscopy, $\mathrm{x}$-ray absorption spectroscopy, $\mathrm{x}$-ray magnetic circular dichroism, and magnetization measurements show that nanoparticles synthesized by thermal decomposition display high crystal quality and bulklike magnetic and electronic properties, while nanoparticles synthesized by coprecipitation show much poorer crystallinity and particlelike phenomenology, including reduced magnetization, high closure fields, and shifted hysteresis loops. The key role of the crystal quality is thus suggested, because particlelike behavior for particles larger than about $5 \mathrm{~nm}$ is observed only when the particles are structurally defective. These conclusions are supported by Monte Carlo simulations. It is also shown that thermal decomposition is capable of producing nanoparticles that, after further stabilization in physiological conditions, are suitable for biomedical applications such as magnetic resonance imaging or biodistribution studies. (C) 2011 American Institute of Physics. [doi:10.1063/1.3559504]
\end{abstract}

\section{INTRODUCTION}

Nanostructured magnetic materials are the basis of a very active research field due to the new phenomena taking place at the nanoscale as a consequence of the interplay of quantum, finite-size, surface, and interfacial effects. Magnetic nanoparticles (NPs) ${ }^{1}$ are an excellent example of nanostructured materials, which provide the critical building blocks for the expansion of many applications of nanotechnology in fields such as biomedicine, ${ }^{2}$ high-density magnetic recording, ${ }^{3}$ and magnetic resonance imaging (MRI). ${ }^{4}$ Key questions in these systems are how the nanostructure modifies their magnetic and electronic properties and how one can take advantage of those new properties to improve the applications. Consequently, understanding and controlling the effects of the nanostructure on the properties of the particles have become increasingly relevant issues for technological applications.

As the size of particles is reduced below about $100 \mathrm{~nm}$, deviations from bulk behavior are widely reported and attributed to changes in the magnetic ordering at the surface layer with respect to that of the particle core, and also to finite-size effects, thus giving rise to a significant decrease in the particle magnetization. ${ }^{1}$ Typically, transition metal oxide NPs, including iron oxide, show surface spin disorder due to symmetry breaking at the surface, leading to ${ }^{5-8}$ (i) a saturation magnetization smaller than in the bulk material, in some cases by a factor of two or more; (ii) high-field differential susceptibility; (iii) extremely high closure fields (even up to

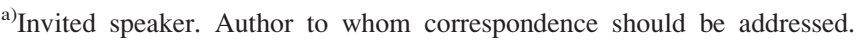
Electronic mail: xavierbatlle@ub.edu.
}

$120 \mathrm{kOe}$ ); (iv) shifted hysteresis loops after field cooling the sample, together with glassy behavior, which has been explained in terms of the existence of a surface layer of disordered spins that freeze in a spin glasslike state due to magnetic frustration, yielding both an exchange field acting on the ordered core and an increase in the particle anisotropy; and (v) extremely high magnetic anisotropy associated with the fact that very high magnetic fields are required to reach magnetic saturation.

However, that particlelike behavior is undesirable in many applications where the NPs should comply with a variety of requirements. In the case of biomedical applications, those include $^{2}$ (i) superparamagnetic behavior at room temperature in order to avoid particle aggregation due to interparticle interactions, (ii) high saturation magnetization to achieve a high magnetic response under the application of moderate magnetic fields, (iii) limiting size for in vivo applications to between 1 and $50 \mathrm{~nm}$, and (iv) biocompatibility and functionality to reach specific targets inside the body. Consequently, biomedical applications require magnetic NPs of a few nanometers in size showing magnetic properties close to those of the bulk material. Research in this field is thus mainly focused on new synthesis methods that enable the preparation of NPs with high crystal quality and reduced magnetic disorder. Iron oxide NPs are suitable candidates due to their low toxicity and ease of functionalization. Magnetite $\mathrm{Fe}_{3} \mathrm{O}_{4}$ and maghemite $\gamma-\mathrm{Fe}_{2} \mathrm{O}_{3}$ are probably among the most widely studied magnetic materials. However, they still garner much attention because several questions remain unanswered. For example, the orbital contribution to the magnetic moment in bulk magnetite is under discussion. ${ }^{9}$ 
In this paper, we report on the effect of the synthesis method and surface coating on the structural quality and magnetic properties of two sets of samples of $\mathrm{Fe}_{3-x} \mathrm{O}_{4} \mathrm{NPs}$ about $5 \mathrm{~nm}$ in size, synthesized by either (i) thermal decomposition of an organic iron precursor in an organic medium, ${ }^{10}$ with a fatty acid (e.g., oleic acid) used as a surfactant covalently bonded to the particle surface $;{ }^{11}$ or (ii) coprecipitation of $\mathrm{Fe}[\mathrm{II}]$ and $\mathrm{Fe}[\mathrm{III}]$ salts in an alkaline aqueous medium, ${ }^{12}$ with polyvinyl alcohol (PVA) used as a protective coating against oxidation adsorbed at the NP surface but without any chemical bond. The crystal quality of both sets of samples determines the occurrence of particlelike phenomenology, which cannot be considered as intrinsic properties arising from finite-size or surface effects.

\section{SAMPLE PREPARATION}

Oleic acid-coated particles $5 \mathrm{~nm}$ in mean diameter were synthesized by thermal decomposition of an iron precursor. ${ }^{10,11}$ One mmol of $\mathrm{Fe}(\mathrm{III})$-acetylacetonate was mixed with $3 \mathrm{mmol}$ of oleic acid, $3 \mathrm{mmol}$ of oleilamine, $5 \mathrm{mmol}$ of 1,2-hexadecanediol, and $10 \mathrm{ml}$ of phenyl ether. The degassed mixture was heated to $200{ }^{\circ} \mathrm{C}$ and kept at that temperature for two hours. Then, it was further heated up to the reflux temperature of phenyl ether $\left(260\right.$ to $\sim 270{ }^{\circ} \mathrm{C}$ ) and kept at that temperature for $30 \mathrm{~min}$. After cooling down to room temperature, the particles were extracted by solvent-nonsolvent techniques and finally resuspended in hexane. Interestingly enough, variations of this procedure enable one to tune the size and shape of the particles. For example, solvents with a higher boiling point lead to larger particles, ${ }^{10}$ and the use of decanoic acid instead of oleic acid as a capping ligand yields particles in a wider range of sizes. ${ }^{13}$ By varying the molar ratio of the decanoic acid to the iron precursor and adjusting the final synthesis temperature, NPs between 5 and $50 \mathrm{~nm}$ are obtained. ${ }^{13}$ The particle size range can be further expanded up to $180 \mathrm{~nm}$ by tuning the heating rate. ${ }^{14}$

PVA-coated NPs $5 \mathrm{~nm}$ in size were prepared by the standard coprecipitation procedure. ${ }^{12} \mathrm{~A}$ stoichiometric mixture of $\mathrm{FeCl}_{3}$ and $\mathrm{FeCl}_{2}$ was dissolved in $10 \mathrm{ml}$ deionized water and added dropwise to $300 \mathrm{ml}$ of a sodium hydroxide aqueous solution containing PVA at room temperature and with a $\mathrm{pH}$ of 10 . The particles formed instantaneously, were extracted by precipitation, and were finally suspended in deionized water at $\mathrm{pH} 7$.

\section{STRUCTURAL CHARACTERIZATION}

High resolution transmission electron microscopy (TEM) images of NPs synthesized from thermal decomposition show regular shapes with very high crystal quality up to the NP surface [Figs. 1(a) and 1(b)]. In contrast, NPs derived from coprecipitation show much more irregular shapes and a lower degree of crystallinity at the surface [Figs. 1(c) and 1(d)], together with in-volume defects. Many of those particles are aggregates of randomly oriented individual crystallites. TEM also shows that the particles prepared by thermal decomposition have a narrow size distribution and are individually coated by the surfactant, while those prepared by

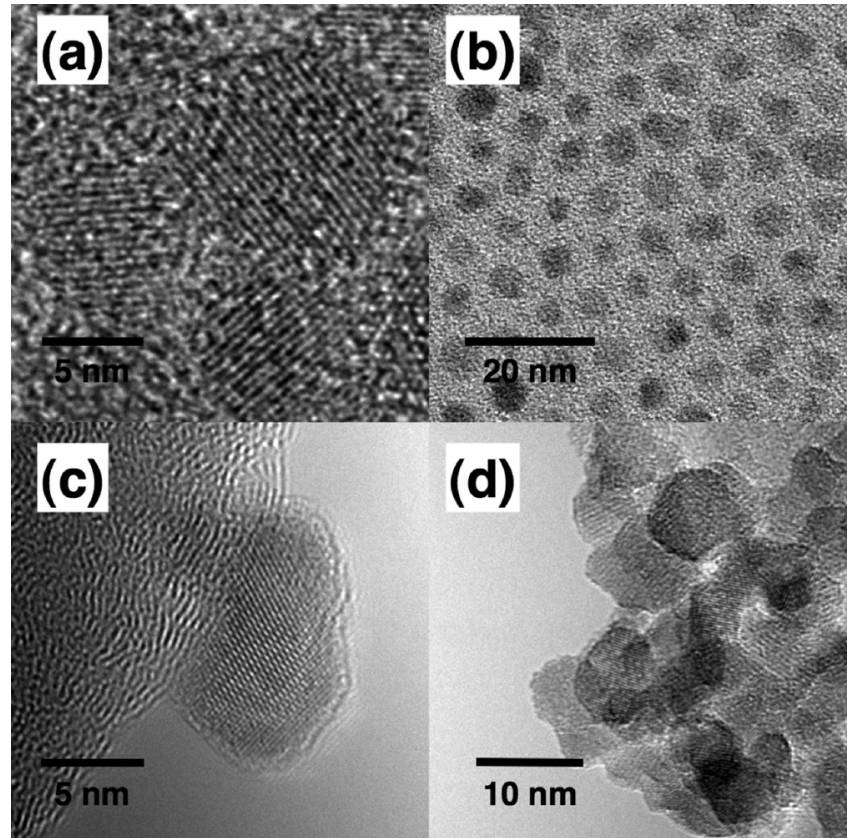

FIG. 1. Oleic acid-coated $\mathrm{Fe}_{3-x} \mathrm{O}_{4}$ NPs prepared by thermal decomposition: [(a) and (b)] high resolution TEM images showing high crystal quality and individual coating by the surfactant. PVA-protected $\mathrm{Fe}_{3-x} \mathrm{O}_{4}$ NPs prepared by coprecipitation: [(c) and (d)] high resolution TEM images showing poorer crystallinity as compared to the previous case and particle aggregation.

coprecipitation tend to agglomerate and show a much broader size distribution.

Figure 2 shows an example of $\mathrm{x}$-ray absorption spectroscopy (XAS) data at the $\mathrm{Fe}_{2,3}$ absorption edges for $\mathrm{Fe}_{3-x} \mathrm{O}_{4}$ NPs about $5 \mathrm{~nm}$ in size. The spectrum for the thermal decomposition particles shows $30 \%$ more holes in the $3 \mathrm{~d}$ band ${ }^{15}$ as compared to the bulk counterpart, ${ }^{9,16}$ indicating an electronic transfer associated with the chemical bonding of the oleic acid at the particle surface. ${ }^{15}$ In contrast, coprecipitation particles of a similar size show an XAS spectrum

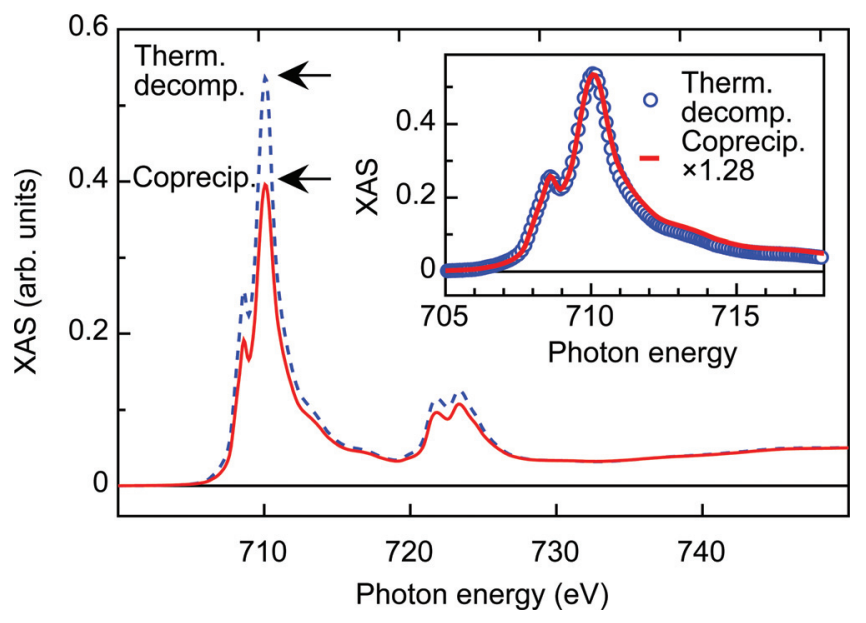

FIG. 2. (Color online) $\mathrm{X}$-ray absorption spectra in the $\mathrm{Fe}^{2,3}$ edges for $\mathrm{Fe}^{3-x} \mathrm{O}^{4}$ NPs about $5 \mathrm{~nm}$ in size, synthesized by thermal decomposition (dashed curve) or coprecipitation (solid curve). Inset: Superimposition of the spectra for the two samples, obtained by multiplying the spectrum of the coprecipitation sample by an appropriate scale factor. 


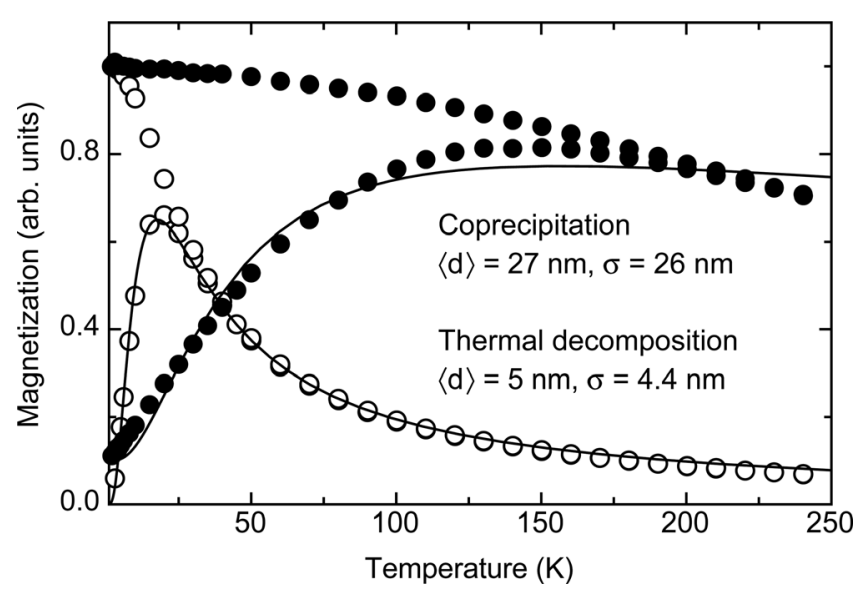

FIG. 3. ZFC-FC curves measured at 50 Oe for $\mathrm{Fe}_{3-x} \mathrm{O}_{4}$ NPs about $5 \mathrm{~nm}$ in size, obtained by thermal decomposition (open circles) or coprecipitation (solid circles). The fits to a distribution of Langevin functions are plotted as solid lines. The diameter $\langle d\rangle$ corresponding to the mean activation magnetic volume and standard deviation $\sigma$ is indicated.

similar to that of the bulk counterpart, as expected for particles with just a protective adsorbed coating. The two spectra can be superimposed almost perfectly, provided that the spectrum for coprecipitation is multiplied by an appropriate scale factor (Fig. 2 inset), which evidences that the stoichiometry is the same within the experimental error. The actual stoichiometry of the $5 \mathrm{~nm}$ NPs synthesized from thermal decomposition was evaluated using Mössbauer spectroscopy and was found to be $\mathrm{Fe}_{2.93} \mathrm{O}_{4},{ }^{17}$ which is compatible with the presence of up to $21 \%$ maghemite, forming an overoxidized surface layer of about one unit cell.

\section{PARTICLELIKE BEHAVIOR VERSUS STRUCTURAL QUALITY}

Magnetization hysteresis loops and zero field coolingfield cooling (ZFC-FC) curves were measured with a superconducting quantum interference device magnetometer. The ZFC-FC curves in Fig. 3 are compared for particles about 5 $\mathrm{nm}$ in size prepared by the two methods. For thermal decomposition, the ZFC curve evidences a very narrow size distribution. The progressive increase of the FC curve below the irreversibility onset indicates the absence of relevant dipolar interactions, which is in agreement with the individual coating of the particles. The coincidence of the onset of the irreversibility and the maximum of the ZFC also confirms that the surfactant coating minimizes the formation of aggregates, avoiding interparticle interactions. In contrast, the ZFC-FC curves for the coprecipitation sample reflect a broader size distribution and particle agglomeration, and the existence of significant interparticle interactions. The mean activation magnetic volume of the two samples was evaluated by fitting the ZFC curves to a distribution of Langevin functions. ${ }^{18}$ The diameter corresponding to the mean activation magnetic volume is $\langle d\rangle=5.0 \mathrm{~nm}$ in the case of thermal decomposition, with a standard deviation of $\sigma=4.4 \mathrm{~nm}$, in very good agreement with the size distribution obtained from TEM. For the coprecipitation sample, $\langle d\rangle=27 \mathrm{~nm}$ and $\sigma=26 \mathrm{~nm}$; these values are much larger than those deduced from the

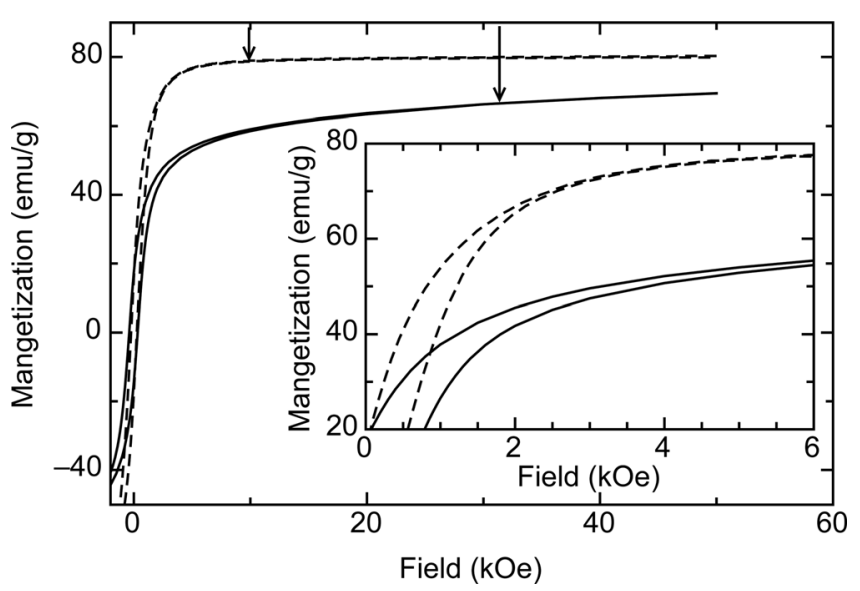

FIG. 4. Magnetization curves at $5 \mathrm{~K}$ for $\mathrm{Fe}_{3-x} \mathrm{O}_{4}$ NPs about $5 \mathrm{~nm}$ in size obtained by thermal decomposition (dashed lines) or coprecipitation (solid lines). The closures of the hysteresis loops are indicated by vertical arrows. Inset: detail of the curves for magnetic fields below $6 \mathrm{kOe}$.

TEM size distribution due to particle aggregation favoring interparticle interactions.

Figure 4 shows the hysteresis loops at $5 \mathrm{~K}$ for particles of about $5 \mathrm{~nm}$. The saturation magnetization is significantly higher for the thermal decomposition sample. Additionally, a higher value of the high-field differential susceptibility and a much more rounded magnetization curve are observed for the coprecipitation sample, facts that are indicative of the presence of magnetic disorder throughout the particles. Moreover, the magnification of the hysteresis curves (Fig. 4 inset) shows the occurrence of much higher closure fields (indicated by arrows in Fig. 4) in the coprecipitation sample, which could be related to the existence of very high energy barriers associated with magnetic frustration induced by structural disorder.

Magnetization values at $5 \mathrm{~T}$ and $5 \mathrm{~K}$ as a function of the particle size are shown in Fig. 5. ${ }^{13,15,19,20}$ The values corresponding to the thermal decomposition samples are always significantly higher than those for coprecipitation, with both sets of data displaying some size dependence. Finite-size and surface effects are evident in the results from Monte Carlo simulations, also shown in Fig. 5 (solid triangles) for a spherical particle with Ising spins on an inverse spinel lattice. ${ }^{21}$ These data have been scaled to the saturation magnetization of bulk magnetite in order to be compared to the experimental results. The experimental values clearly lie below the ones obtained by Monte Carlo simulation, even for thermal decomposition samples. This could be due to the difficulty in obtaining pure, stoichiometric magnetite without a significant amount of the maghemite phase for particle sizes below a few tens of nanometers. In the case of coprecipitation, the lack of crystal quality yields a large, additional reduction in the magnetization due to magnetic disorder within the particle.

$\mathrm{X}$-ray magnetic circular dicroism (XMCD) was measured at the $\mathrm{Fe}_{2,3}$ absorption edges for $\mathrm{Fe}_{3-x} \mathrm{O}_{4}$ NPs about 5 $\mathrm{nm}$ in size. ${ }^{15}$ The measurements were carried out with total electron yield detection and an applied field of $2 \mathrm{~T}$ parallel to the x-ray beam. The application of magneto-optical sum 


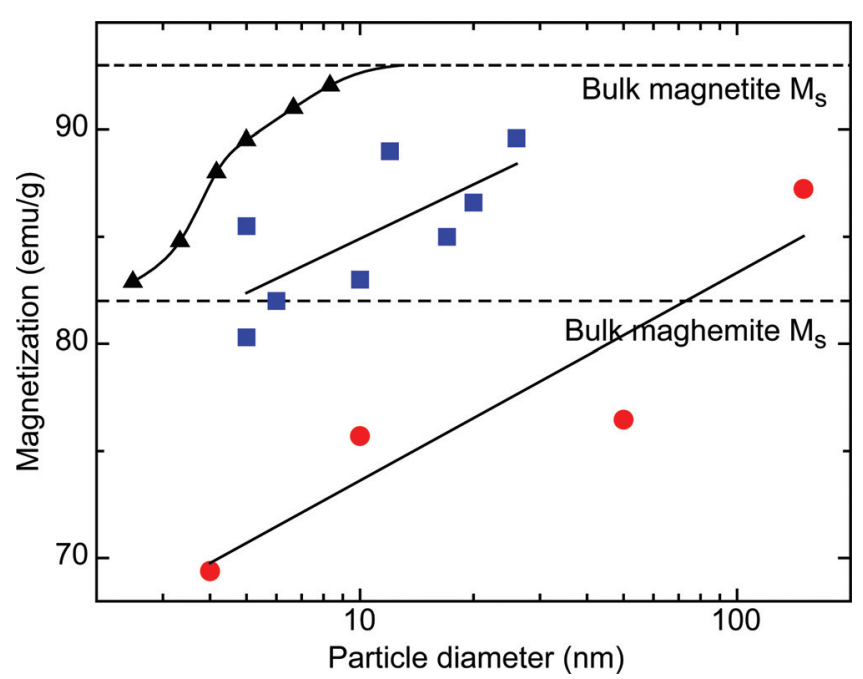

FIG. 5. (Color online) Magnetization as a function of the particle diameter for $\mathrm{Fe}_{3-x} \mathrm{O}_{4}$ NPs synthesized by coprecipitation (solid circles) or thermal decomposition (solid squares) at $5 \mathrm{~T}$ and $5 \mathrm{~K}$. Spontaneous magnetization values were obtained from Monte Carlo simulations and scaled to the saturation magnetization of bulk magnetite (solid triangles). The saturation magnetizations $M_{s}$ for bulk magnetite and maghemite are indicated as dashed horizontal lines. Solid lines are guides for the eye.

rules on the XMCD spectra enables one to obtain the magnetic moment per formula unit, $\mu_{\mathrm{Fe}}$, and the spin and orbital contributions $^{22}$ (Table I). The total magnetic moment per formula unit for the thermal decomposition sample is about $42 \%$ larger than that for the coprecipitation sample, in excellent agreement with magnetization results. Furthermore, $\mu_{\mathrm{Fe}}$ for the thermal decomposition sample is just about $16 \%$ smaller than that reported from previous XMCD measurements on a magnetite single-crystal, ${ }^{9}$ which is also in excellent agreement with magnetization results. The calculated orbital magnetic moments are 0.036(8) and 0.081(9) $\mu_{\mathrm{B}} / \mathrm{f}$.u. for the thermal decomposition and coprecipitation samples, respectively. Although both values are in qualitative agreement with Ref. 9, the coprecipitation sample shows about a threefold increase with respect to the expected value of the ratio of the orbital-to-spin angular moments $\mathrm{m}_{\mathrm{L}} / \mathrm{m}_{\mathrm{S}} \approx 0.013$, obtained from Local Density Approximation calculations for bulk magnetite. ${ }^{23}$ Increased values of $\mathrm{m}_{\mathrm{L}} / \mathrm{m}_{\mathrm{S}}$ are usually found in low dimensional and particlelike systems. ${ }^{24}$ In contrast, the thermal decomposition sample fully recovers the value for the bulk orbital-to-spin moment, which reinforces the idea that the high crystal quality of the sample is behind the observed bulklike magnetic and electronic behavior. ${ }^{15}$

In order to elucidate the influence of the crystal quality and particle/surfactant chemical bond of the thermal decomposition samples on the magnetic disorder at the NP

TABLE I. Total number of $3 \mathrm{~d}$ holes, $N_{h}$; magnetic moment per formula unit, $\mu_{L}$ (orbital), $\mu_{S}$ (spin), and $\mu_{\mathrm{Fe}}$ (total); and orbital-to-spin moment ratio, $\mathrm{m}_{\mathrm{L}} / \mathrm{m}_{\mathrm{S}}$, for oleic acid and PVA samples.

\begin{tabular}{lccccc}
\hline \hline Sample & $N_{h}$ & $\mu_{\mathrm{L}}\left(\mu_{\mathrm{B}}\right)$ & $\mu_{\mathrm{S}}\left(\mu_{\mathrm{B}}\right)$ & $\mu_{\mathrm{Fe}}\left(\mu_{\mathrm{B}}\right)$ & $\mathrm{m}_{\mathrm{L}} / \mathrm{m}_{\mathrm{S}}(\%)$ \\
\hline Thermal decomposition & $17.5(3)$ & $0.036(8)$ & $3.24(5)$ & $3.27(6)$ & $1.1(3)$ \\
Coprecipitation & $13.7(3)$ & $0.081(9)$ & $2.25(3)$ & $2.31(3)$ & $3.6(4)$ \\
\hline \hline
\end{tabular}

TABLE II. Fitted values of the volume and surface anisotropy constants, $K_{v}$ and $K_{s}$, respectively, for thermal decomposition samples.

\begin{tabular}{|c|c|c|}
\hline & Fitted & Expected \\
\hline$K_{v}$ & $2.4 \times 10^{5} \mathrm{erg} \mathrm{cm}^{3}$ & $2.2 \times 10^{5} \mathrm{erg} \mathrm{cm}^{3}$ (below the Verwey transition) \\
\hline$K_{S}$ & $0.029 \mathrm{erg} \mathrm{cm}^{-2}$ & $0.02-0.04 \mathrm{erg} \mathrm{cm}^{-2}$ \\
\hline
\end{tabular}

surface, we evaluated the surface and volume anisotropies ( $K_{S}$ and $K_{v}$, respectively) from the size distribution obtained from TEM and $T \ln \left(t / \tau_{0}\right)$ scaling of the thermoremanence relaxation data. ${ }^{7,8,25,26}$ In the absence of sizable interparticle interactions, as is the case for the thermal decomposition particles (see Fig. 3), the derivative of the $T \ln \left(t / \tau_{0}\right)$ scaling yields the energy barrier distribution associated with the effective anisotropy for magnetization reversal. Assuming the ad hoc expression for the effective, uniaxial anisotropy, $K_{\text {eff }}=K_{v}+(6 / D) K_{s}$, the size distribution obtained from TEM can be superimposed onto the energy barrier distribution by a fitting procedure that yields the volume and surface anisotropies. ${ }^{27}$ The values obtained are given in Table II, and are in very good agreement with other experimental values (see Refs. 27 and 28, and references therein). Thus, intrinsic surface effects, rather than the lack of crystallinity, dominate the surface anisotropy in particles with high crystal quality on the nanometer scale.

Another effect usually related to magnetic disordered systems is the occurrence of shifted magnetization hysteresis loops, which in many cases is explained by assuming the existence of the exchange bias phenomenon. ${ }^{29,30}$ Shifted loops can be observed after FC processes, or even ZFC, for samples prepared by coprecipitation, ${ }^{19,31}$ as shown in Fig. 6 for $5 \mathrm{~nm} \mathrm{Fe}{ }_{3-x} \mathrm{O}_{4}$ PVA-coated NPs having poor crystallinity. Additionally, hollow maghemite particles $8 \mathrm{~nm}$ in size and composed of about 10 randomly oriented crystallites show strongly shifted loops and very high closure fields due to their highly defective crystallographic structure and the large interface areas among single crystallites. ${ }^{32}$ All those systems have in common the occurrence of magnetic disorder associated with defective crystal structures, giving rise to high energy barriers that can be overcome only at very high fields.

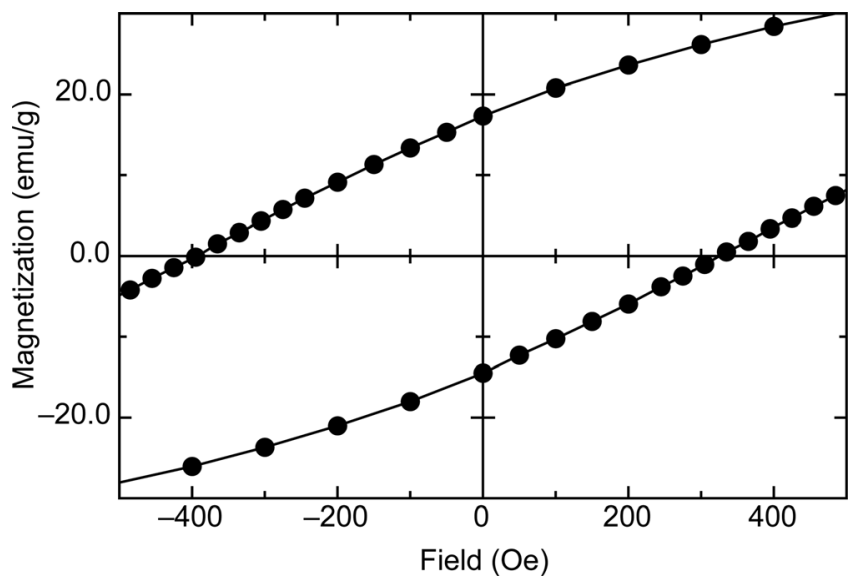

FIG. 6. Detail of the hysteresis loop at $5 \mathrm{~K}$ for $5 \mathrm{~nm} \mathrm{Fe}{ }_{3-x} \mathrm{O}_{4}$ PVA-coated NPs synthesized by coprecipitation, measured after field cooling the sample at $2 \mathrm{kOe}$, showing loop shift to negative fields. 


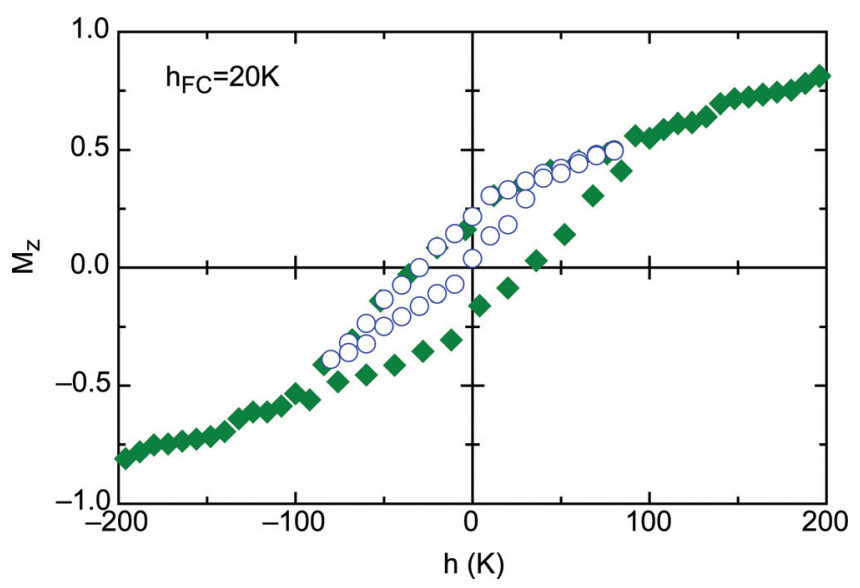

FIG. 7. (Color online) Atomistic, Monte Carlo simulation of two magnetization $\left(M_{z}\right)$ hysteresis cycles at $0.5 \mathrm{~K}$ of a spherical maghemite particle 4 unit cells in diameter with $40 \%$ vacancies at surface Fe positions. The particle was field cooled (FC) at a reduced field of $20 \mathrm{~K}$, and the maximum field applied in the cycles was either $80 \mathrm{~K}$ (open circles) or $200 \mathrm{~K}$ (solid squares).

In contrast, thermal decomposition samples with high crystal quality do not show this phenomenology at all. For very defective particles showing high closure fields (Fig. 4 inset), the applied magnetic field of a FC process imprints an effective anisotropy that cannot be erased at the maximum applied field of the hysteresis loop. Therefore, the system follows a minor loop that, in some cases, has been attributed to an exchange coupling phenomenon between a core-shell structure. ${ }^{29,31,33}$

In order to reinforce this interpretation, Fig. 7 shows two hysteresis loops obtained by atomistic Monte Carlo simulations of a single spherical maghemite particle 4 unit cells in diameter, with $40 \%$ of the vacancies in the Fe positions at the surface. ${ }^{21}$ Both magnetization curves were obtained after FC down to $0.5 \mathrm{~K}$ in a field of $h_{\mathrm{FC}}=20 \mathrm{~K}$ (the field is given in temperature units as $\mu H / k_{B}$ ), while the maximum applied field was $80 \mathrm{~K}$ in one case and $200 \mathrm{~K}$ in the other. It is obvious that the apparent shifted loop in the case of $h=80 \mathrm{~K}$ is just a minor loop of that corresponding to $h=200 \mathrm{~K}$, because it was obtained for a maximum field lower than the closure field (approximately $90 \mathrm{~K}$ ). Similar apparent shifted loops, which in fact are minor loops, have also been reported. ${ }^{32}$

\section{MAGNETITE NANOPARTICLES FOR BIOMEDICAL APPLICATIONS}

As iron oxides are approved for biomedical use in humans due to their low toxicity, magnetite is the material of choice when the highest signal is desired, because its saturation magnetization per unit volume is about $14 \%$ higher than that of maghemite. We have shown that thermal decomposition of an organic Fe precursor is an appropriate method to obtain magnetite NPs with high structural and magnetic quality. However, this chemical route yields hydrophobic particles that require further functionalization and stabilization under physiological conditions. This implies surface modification by attaching specific ligands and/or coating with inorganic shells. As noted above, the use of decanoic acid as a capping ligand yields particles of regular shapes, with high crystal quality up to the surface, in a wide range of sizes. ${ }^{13}$ The relatively short hydrocarbon chain of decanoic acid makes these NPs dispersible in slightly polar organic media, such as alcohols. This is an advantage when further coating of the particles is required, because usually that reaction takes place in polar solvents, as in the case of $\mathrm{SiO}_{2}$ coating by the Stöber method. ${ }^{34}$

On the other hand, when oleic acid is used as a capping ligand, it is possible to ligand exchange the NPs to dimercaptosuccinic acid (DMSA), which renders the particle surface hydrophilic. ${ }^{35}$ Although the ligand exchange process reduces the saturation magnetization in about $10 \%$ due to some surface oxidation and promotes the formation of a small amount of aggregates constituted of a few particles, the magnetic quality is still much higher than that of the NPs prepared by coprecipitation. $^{36}$ These DMSA-coated NPs display MRI relaxivity values higher than those of commercially available contrast agents. Moreover, it also is possible to obtain highcontrast MRI images of the livers and brains of mice by injecting the particles intravenously and making them overcome the blood-brain barrier by osmotic disruption. ${ }^{36}$ The high magnetic signal of these particles enabled us to carry out a biodistribution study by means of magnetization measurements, which could be an excellent alternative to the usual histograms obtained from microscopy. The magnetization for samples of lyophilized mouse liver, kidney, and spleen was measured and showed the presence of diamagnetic material (tissue), paramagnetic natural ferritin, and superparamagnetic particles. The comparison of the signal of the samples extracted from inoculated animals to those of control animals allowed for accurate determination of the percentage of internalization of the NPs with respect to the total dose, leading to $35 \%$ for liver, $1.5 \%$ for spleen, and $0.5 \%$ for kidney. ${ }^{36}$

\section{SUMMARY AND CONCLUSIONS}

We have shown that in magnetic NPs, bulklike behavior is strongly tied to the crystal quality of the samples. When particles are larger than about $5 \mathrm{~nm}$ in size, particlelike behavior, including strongly reduced magnetization, high values of the closure fields, and shifted loops, are observable only in NPs with poor crystallinity. This is supported by Monte Carlo simulation showing that intrinsic finite-size and surface effects ${ }^{1}$ are relevant only for sizes below about $5 \mathrm{~nm}$. We have also shown that thermal decomposition is a very versatile method to produce high quality nanoparticles between 5 and $50 \mathrm{~nm}$ in size with bulklike properties. These particles are also suitable for biomedical applications. All in all, these results suggest the key role of the crystal quality in the magnetic and electronic properties of ferromagnetic NPs and, in particular, the fact that, in many cases, the magnetic disorder phenomena observed in single-phase particles larger than a few nanometers in diameter should not be considered as an intrinsic effect associated with the finite size.

\section{ACKNOWLEDGMENTS}

Funding from the Spanish MICINN (MAT2009-08667, MAT2008-01077, and CSD2006-00012), Catalan DIUE (2009SGR856), and Aragonese DGA (CAMRADS and IMANA) is acknowledged. 
${ }^{1}$ X. Batlle and A. Labarta, J. Phys. D: Appl. Phys. 35, R15 (2002). ${ }^{2}$ P. Tartaj, M. P. Morales, S. Veintemillas-Verdaguer, T. Gonzalez Carreno, and C. J. Serna, J. Phys. D: Appl. Phys. 36, R182 (2003).

${ }^{3}$ T. Hayashi, S. Hirono, M. Tomita, and S. Umemura, Nature 381, 772 (1996).

${ }^{4}$ J. Kim, S. Park, J. E. Lee, S. M. Jin, J. H. Lee, I. S. Lee, I. Yang, J.-S. Kim, S. K. Kim, M.-H. Cho, and T. Hyeon, Angew. Chem., Int. Ed. 45, 7754 (2006).

${ }^{5}$ X. Batlle, X. Obradors, M. Medarde, J. Rodríguez-Carvajal, M. Pernet, and M. Vallet, J. Magn. Magn. Mater. 124, 228 (1993).

${ }^{6}$ X. Batlle, M. García del Muro, J. Tejada, H. Pfeiffer, P. Görnert, and E. Sinn, J. Appl. Phys. 74, 3333 (1993).

${ }^{7}$ X. Batlle, M. García del Muro, and A. Labarta, Phys. Rev. B 55, 6440 (1997).

${ }^{8}$ M. García del Muro, X. Batlle, and A. Labarta, Phys. Rev. B 59, 13584 (1999).

${ }^{9}$ E. Goering, S. Gold, M. Lafkioti, and G. Schütz, Europhys. Lett. 73, 97 (2006); E. Goering, M. Lafkioti, and S. Gold, Phys. Rev. Lett. 96, 039701 (2006).

${ }^{10}$ J. Park, K. An, Y. Hwang, J.-G. Park, H.-J. Noh, J.-Y. Kim, J.-H. Park, N.-M. Hwang, and T. Hyeon, Nature Mater. 3, 891 (2004).

${ }^{11}$ A. G. Roca, M. P. Morales, K. O'Grady, and C. J. Serna, Nanotechnology 17, 2783 (2006).

${ }^{12}$ R. Massart, IEEE Trans. Magn. 17, 1247 (1981).

${ }^{13}$ P. Guardia, N. Pérez, A. Labarta, and X. Batlle, Langmuir 26, 5843 (2010).

${ }^{14}$ P. Guardia, J. Pérez-Yuste, A. Labarta, X. Batlle, and L. Liz-Marzán, Chem. Commun. (Cambridge) 46, 6108 (2010).

${ }^{15}$ N. Pérez, F. Bartolomé, L. M. García, J. Bartolomé, M. P. Morales, C. J. Serna, A. Labarta, and X. Batlle, Appl. Phys. Lett. 94, 093108 (2009).

${ }^{16}$ Unpublished data courtesy of J. C. Cèzar (ESRF), March 13, 2008.

${ }^{17}$ A. G. Roca, J. F. Marco, M. P. Morales, and C. J. Serna, J. Phys. Chem. C 111, 18577 (2007).

${ }^{18}$ J. L. Dormann, D. Fiorani, and E. Tronc, Adv. Chem. Phys. 98, 283 (1997).

${ }^{19}$ G. F. Goya, T. S. Berquo, F. C. Fonseca, and M. P. Morales, J. Appl. Phys. 94, 3520 (2003).
${ }^{20}$ P. Guardia, B. Batlle-Brugal, A. G. Roca, O. Iglesias, M. P. Morales, C. J. Serna, A. Labarta, and X. Batlle, J. Magn. Magn. Mater. 316, e756 (2007).

${ }^{21}$ O. Iglesias and A. Labarta, Phys. Rev. B 63, 184416 (2001).

${ }^{22}$ B. T. Thole, P. Carra, F. Sette, and G. van der Laan, Phys. Rev. Lett. 68, 1943 (1992).

${ }^{23}$ H.-T. Jeng and G. Y. Guo, Phys. Rev. B 65, 094429 (2002).

${ }^{24}$ J. Bartolomé, F. Bartolomé, L. M. García, G. Filoti, T. Gredig, C. N. Colesniuc, I. K. Schuller, and J. C. Cezar, Phys. Rev. B 81, 195405 (2010); J. Bartolomé, F. Bartolomé, L. M. García, E. Roduner, Y. Akdogan, F. Wilhelm, and A. Rogalev, ibid. 80, 014404 (2009).

${ }^{25}$ A. Labarta, O. Iglesias, Ll. Balcells, and F. Badia, Phys. Rev. B 48, 10240(1993)

${ }^{26}$ O. Iglesias, F. Badia, A. Labarta, and Ll. Balcells, Z. Phys. B 100, 173 (1996)

${ }^{27}$ N. Pérez, P. Guardia, A. G. Roca, M. P. Morales, C. J. Serna, O. Iglesias, F. Bartolome, L. M. Garcia, X. Batlle, and A. Labarta, Nanotechnology 19, 475704 (2008).

${ }^{28}$ R. A. Lefever, "Magnetic and other properties of oxides and related compounds: Spinels, Fe oxides, and Fe-Me-O compounds," in Landolt-Börnstein: Numerical Data and Functional Relationships in Science and Technology-New Series, edited by K. H. Hellwege and A. M. Hellwege (Springer, Berlin, 1980), Vol. III/12b, pp. 61-62.

${ }^{29}$ B. Martinez, X. Obradors, L. Balcells, A. Rouanet, and C. Monty, Phys. Rev. Lett. 80, 181 (1998).

${ }^{30}$ S. A. Makhlouf, H. Al-Attar, and R. H. Kodama, Solid State Commun. 145, 1 (2008).

${ }^{31}$ K. Tao, H. Dou, and K. Sun, Chem. Mater. 18, 5273 (2006).

${ }^{32}$ A. Cabot, A. P. Alivisatos, V. F. Puntes, L. Balcells, O. Iglesias, and A. Labarta, Phys. Rev. B 79, 094419 (2009).

${ }^{33}$ O. Iglesias, A. Labarta, and X. Batlle, J. Nanosci. Nanotechnol. 8, 2761 (2008).

${ }^{34}$ W. Stöber, A. Fink, and A. Bohm, J. Colloid Interface Sci. 26, 62 (1968).

${ }^{35}$ A. G. Roca, S. Veintemillas-Verdaguer, M. Port, C. Robic, C. J. Serna, and M. P. Morales, J. Phys. Chem. B 113, 7033 (2009).

${ }^{36}$ R. Mejías, S. Pérez-Yagüe, A. G. Roca, N. Pérez, Á. Villanueva, M. Cañete, S. Mañes, J. Ruiz-Cabello, M. Benito, A. Labarta, X. Batlle, S. Veintemillas-Verdaguer, M. P. Morales, D. F. Berber, and C. J. Serna, Nanomedicine 5, 397 (2010). 